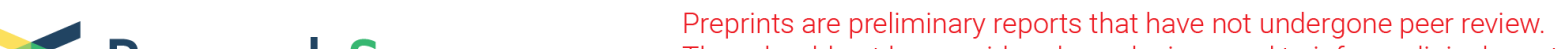 $\begin{array}{ll}\text { Research Square } & \text { They should not be considered conclusive, used to inform clinical practice, } \\ \text { or referenced by the media as validated information. }\end{array}$
}

\section{$\beta$-Amyloid Protein Induces Mitophagy-Dependent Ferroptosis Through the CD36/PINK/PARKIN Pathway Leading to Blood-Brain Barrier Destruction in Alzheimer's Disease}

\section{li Jianhua}

Huazhong University of Science and Technology

\section{Li mengyu}

Huazhong University of Science and Technology

\section{Ge Yangyang}

Huazhong University of Science and Technology

Chen Jiayi

Huazhong University of Science and Technology

Ma Jiamin

Huazhong University of Science and Technology

\section{Wang Chenchen}

Huazhong University of Science and Technology

\section{Sun Miaomiao}

Huazhong University of Science and Technology

\section{Wang Li}

Huazhong University of Science and Technology

\section{Yao shanglong}

Huazhong University of Science and Technology

yao chengye ( $\square$ yaochengye@hust.edu.cn )

Huazhong University of Science and Technology

\section{Research}

Keywords: Alzheimer's disease, BBB, pericytes, fatty acid transporter, mitophagy, ferroptosis

Posted Date: December 9th, 2021

DOI: https://doi.org/10.21203/rs.3.rs-1087555/v1

License: (c) (1) This work is licensed under a Creative Commons Attribution 4.0 International License. Read Full License 
Page 2/31 


\section{Abstract \\ Background}

Blood-brain barrier (BBB) dysfunction may occur in the onset of Alzheimer's disease (AD). While pericytes are a vital part of the neurovascular unit and the BBB, acting as the gatekeeper of the BBB. Amyloid $\beta$ $(A B)$ deposition and neurofibrillary tangles in the brain are the central pathological features of AD. CD36 promotes vascular amyloid deposition and leads to vascular brain damage, neurovascular dysfunction, and cognitive deficits. However, the molecular mechanism in destroying pericytes of the BBB are still unclear.

\section{Objectives}

To investigate the effect of low-dose Aß1-40 administration on pericyte outcome and BBB injury molecular mechanism.

\section{Methods}

We selected 6-month-old and 9-month-old APP/PS1 mice and wild-type (WT) mice of the same strain, age, and sex as controls. We assessed the BBB by PET/CT. Brain pericytes were extracted and cocultured with endothelial cells (bEnd.3) to generate an in vitro BBB model to observe the effect of AB1-40 on the BBB. Furthermore, we explored the intracellular degradation and related molecular mechanisms of A 1-40 after being engulfed in cells through CD36.

\section{Results}

BBB permeability and the number of pericytes decreased in APP/PS1 mice. AB1-40 increases the permeability of the BBB in an in vivo model and downregulates the expression of $C D 36$, which reversed the $A \beta$-induced changes in $B B B$ permeability. A $1-40$ was phagocytized in pericytes with high expression of CD36. We observed that this molecule inhibited pericyte proliferation, caused mitochondrial damage, and increased mitophagy. Finally, we confirmed that A 1-40 induced pericyte mitophagy-dependent ferroptosis through the CD36/PINK1/Parkin pathway.

\section{Conclusions}

PDGFR $\beta$ (a marker of pericytes), CD36, and amyloid $\beta$ colocalized in vitro and in vivo and that $A \beta 1-40$ caused BBB destruction by upregulating the expression of CD36 in pericytes. The mechanism by which A 1-40 destroys the BBB involves induction of pericyte mitophagy-dependent ferroptosis through the CD36/PINK1/Parkin pathway. 


\section{Introduction}

More than $50 \%$ of clinically diagnosed Alzheimer's disease (AD) patients have vascular alterations, but the role of age-related vascular factors in the mechanism of AD is still unclear [1]. Before the onset of dementia, neurodegeneration, and/or atrophy, initial blood-brain barrier (BBB) impairment and/or dysfunction can be observed in $A D$ [2-5]. Particularly at the capillary level, pericytes play a vital role in the formation and maintenance of the BBB [6-8]. Pericytes degenerate in individuals with $A D$ and play a crucial role in the $B B B$ clearance of $A B$ [9-11]. Pericyte death occurs when $A \beta$ aggregates in the cell, exceeding the clearance of this protein, or when hypoxia occurs. Transgenic mice deficient in PDGFR $\beta$ in pericytes not only have learning and memory impairments but also have enhanced $A \beta$ deposition in the brain and neuronal cell death in the hippocampus and cerebral cortex $[12,13]$. Pericytes may transport $A \beta$ across the BBB into the blood $[14,15]$. Previously, the role of endothelial cells in the BBB has been extensively researched, but there have been few studies on the role of pericytes in the destruction of the BBB by $A \beta$. Studies on the changes in pericytes due to $A \beta$ are lacking.

$A \beta$ is formed by amyloid precursor protein (APP) [16], a membrane protein that acts as a signal receptor during neuronal activity. Its production and accumulation in the brain of individuals with $A D$ are considered the hallmark of the amyloid hypothesis [17]. A typical cerebrovascular disease associated with $A \beta 1-40$ deposition is cerebral amyloid angiopathy (Aß-cerebral amyloid angiopathy, CAA) [18]. The destruction of the BBB is an essential reason for the peripheral clearance of $A \beta$ [19]. This phenomenon not only decreases the release of $A \beta$ but also increases the transport of peripheral $A \beta$ to the center through the BBB, which eventually leads to high levels of intracranial $A \beta$ deposition [20], accelerating disease progression. The mechanism of $A \beta$-induced $B B B$ destruction is still unclear. In fact, $A \beta$ is the initiating factor of BBB destruction. Autopsy results showed apparent BBB destruction around blood vessels with $A B$ deposition. Therefore, elucidation of the mechanism of $A \beta$ injury to the $B B B$ will help maintain the integrity of the BBB and decrease $A \beta$ deposition in the central nervous system.

Fatty acid transporter, also known as scavenger receptor cluster 36 (CD36), is a multifunctional glycoprotein involved in apoptotic cell uptake, signal transduction, cell adhesion, angiogenesis, and immune function [21, 22]. This molecule is essential for $A \beta 1-40$-induced vascular oxidative stress and neurovascular dysfunction. CD36 can also change its structure to phagocytose and absorb cellular contents such as oxidized phospholipids, apoptotic cells, and amyloid [23,24]. Previous studies have confirmed that the lack of CD36 can also offset the cerebrovascular dysfunction of Tg2576 mice and partially normalize the number and morphology of pericytes [25]. However, its expression and mechanism after low-dose AB1-40 stimulation of pericytes are still unclear. In particular, the role of CD36 in pericytes in the BBB may become a new molecular target for the treatment of AD-related amyloid angiopathy.

\section{Materials And Methods}

\subsection{Animals}


6-month-old and 9-month-old APP1/PS1 double transgenic AD model mice (9 each) [provided by Jiangsu Changzhou Cavens Experimental Co., Ltd. (license number: SCXK (Su) 2016-0010)] and 9 WT mice (C57BL/6J) were used [provided by Beijing Sibefu Biotechnology Co., Ltd. (license number: SCXK (Jing) 2019-0010)]. The animals were kept in an SPF animal room at a constant temperature $\left(23^{\circ} \mathrm{C} \pm 2^{\circ} \mathrm{C}\right)$, and the conditions were adjusted: alternation of white light and dark (8:00 in the morning-8:00 in the white evening light; 8:00 in the evening-8:00 in the morning) and free access to food and water. The mice used in this experiment were all males, which met the SPF clean-level standard for laboratory animals issued by the Ministry of Health. Male Sprague-Dawley (SD) rats (30-550 g, approximately 3 weeks old) were provided by the Animal Experiment Center of Tongji Medical College, Huazhong University of Science and Technology. During the experiment, raising and obtaining animal materials complied with the relevant rules and regulations of the Animal Management Committee of Huazhong University of Science and Technology and the International Association for Animal Research (IASP) on the management and protection of experimental animals.

\subsection{Antibodies and reagents}

The following antibodies were used in this study: $\beta$-amyloid (20.1) (Santa Cruz Biotechnology), PDGFR $\beta$ (Abcam), NG2 (Abcam), LRP1 (Abcam), CD36 (ABclonal), CD31 (CST), GLUT1 (Abcam), CD36 (BD), $\beta$ actin (ABclonal), HSP60 (Santa Cruz Biotechnology), Tim23 (ABclonal), LC3B (Sigma-Aldrich), cleavedcaspase 3 (CST), caspase 3 (ABclonal), BAX (ABclonal), BCL2 (ABclonal), GPx4 (Proteintech), xCT (Proteintech), NOX1 (Proteintech), GAPDH (Proteintech), ferritin (Beyotime), and a Mitophagy Antibody Sampler Kit (CST). Aß1-40 (ChinaPeptides Co., Ltd.), BODZPY 581/591 (Invitrogen), Mito-FerroGreen (Dojindo), LysoTracker Red (Invitrogen), HiLyte Fluor ${ }^{T M}$ 555-Aß1-40 (Anaspec), FITC-Aß1-40 (Bachem), and FITC-dextran (Sigma-Aldrich) were used.

\subsection{Mouse brain PET/CT imaging}

Abnormal changes in brain $18 \mathrm{~F}$ fluorodeoxyglucose (18F-FDG) uptake are often used as a sensitive indicator of the BBB [26]. The procedure used was as follows: 1 . One day before the experiment, the mice had not fasted with food and water; 2 . Gas anesthesia ( $2 \%$ isoflurane) and tail vein injection of $200 \pm 10$ $\mu \mathrm{Ci} 18 \mathrm{~F}-\mathrm{FDG}$ were used; 3 . Then, scanning was immediately started, PET was first performed, followed by CT; 4. PET was performed as follows: dynamic mode, head scan for $10 \mathrm{~min}$, and CT was performed as follows: normal mode; 5 . The reconstruction conditions were as follows: PET: two iterations, 12 subsets, slightly filtered; time division: 1 min*10 CT: FDK, image size: 256, FOV: 1.0;6. After scanning, the mice were placed in a lead-shielded room, restored to a regular diet, and fed normally after $1018 \mathrm{~F}$ half-lives.

\subsection{Brain sampling}

We use $4 \%$ sodium pentobarbital [50 mg/ $\mathrm{kg}$, intraperitoneal (i.p.)] to anesthetize the mice and then fixed them on the operating table. After thorough disinfection with $75 \%$ alcohol, tissue scissors were used to cut the skin along the midline of the mouse sternum from bottom to top. The sternum was cut to fully expose the heart. Care was taken to prevent damage the blood vessels. After the diaphragm was carefully broken, toothless long forceps were used. After carefully clamping and fixing the heart, we quickly 
inserted the perfusion needle from the apex of the heart into the left ventricle, paying attention to the angle and depth of the needle to avoid penetrating the interventricular septum. Then, we cut a small opening in the right atrial appendage with ophthalmological scissors, opened the perfusion valve, infused precooled saline from the left ventricle, and allowed flow out from the right atrial appendage through the large circulation; the outflow was colorless. The lungs, liver, and mucous membranes were whitish. The mice were perfused and fixed with $4 \%$ paraformaldehyde at $4^{\circ} \mathrm{C}$ for approximately $10 \mathrm{~min}$. Finally, the intact brain tissue was carefully removed and soaked in $4 \%$ paraformaldehyde for histological analysis.

\subsection{Immunofluorescence of brain slices.}

Brain slices prepared from the indicated groups of mice were subjected to double immunofluorescence staining. The primary antibodies used in our studies were mouse anti- $\beta$-amyloid (20.1) (1:25, Santa Cruz Biotechnology), rabbit anti-PDGFR 3 (1:100, Abcam), and rabbit anti-CD36 (1:100 ABclonal). All images were obtained, and colocalization was visualized by using a Nikon confocal microscope.

\subsection{Cell isolation, generation, culture, and activation.}

In this experiment, the bEnd. 3 cell line was purchased from ATCC and was passaged once in 3-4 days according to the company's requirements. Brain pericytes were extracted according to a previous extraction method $[27,28]$ and improved. Each time, 5 male SD rats were used for approximately 3 weeks (30-50 g). The SD rats were from Huazhong provided by the Animal Experiment Center, Tongji Medical College, University of Science and Technology. After anesthetizing the rat (the method was the same as before), we immediately removed the brain. Then, the whole brain was placed in prechilled $75 \%$ alcohol for 3-5 min for disinfection, the cerebral cortex was removed and $500 \mu$ l each of $0.1 \%$ type $\nabla$ collagenase and DNase I $(1000 \mathrm{U} / \mathrm{ml})$ was added and the brain tissue was digested at a constant temperature water bath at $37^{\circ} \mathrm{C}$ for approximately 1 hour. Finally, the capillaries were separated with Percoll and seeded in a 6-well plate containing a unique medium for pericytes. After 72 hours, the medium was changed for the first time, and then, a new medium was added every 2 days. PDGFR- $\beta$ and CD31 antibodies were used for immunostaining identification.

\subsection{A $\mathrm{\beta}$ uptake}

Primary cells were incubated with $500 \mathrm{nM}$ human HyLyte Fluor ${ }^{\mathrm{TM}}$ 555-labeled A $\mathrm{A} 1-40$ (Anaspec, Fremont, $\mathrm{CA}$ ) for 2 hours in serum-free media as previously described [29]. Following treatment, the cells were rinsed twice with 1X PBS without calcium and magnesium. We observed the fluorescence associated with $A \beta$ through a fluorescence microscope and superimposed the cells under white light to indicate the phagocytosis of $A \beta$. The cells on the slides were fixed and processed for imaging according to the abovementioned protocol for immunocytochemistry. After incubation with the primary anti-CD36 antibody (1:200), the cells were stained with DAPI to observe the colocalization of CD36 and AB1-40.

\subsection{Construction of in vitro BBB model}

After separate culture of the BBB model, bEnd. 3 cells $(1.0 \times 105$ cells $/ \mathrm{cm} 2)$ were placed on the front side of the Transwell and then cultured. For the contact cocultivation model, we referred to a previous method 
$[30,31]$. Transwell cell membranes $(24$ wells, $0.4 \mu \mathrm{m})$ were seeded with pericytes $(1.0 \times 104 \mathrm{cells} / \mathrm{cm} 2)$ on the reverse side. After 6 hours, the pericytes were firmly attached, and then, bEnd. 3 cells $(1.0 \times 105$ cells/cm2) were seeded on the front side of the Transwell cell membrane and cultured. Pericytes and bEnd. 3 cells were in contact through the small holes between the membranes. Generally, the fluid was changed every 2 days, and the BBB model was established for use in approximately 6 days.

\section{9 (Trans-endotheilal electrical resistance, TEER) determination}

The TEER was measured with the EVOM2 resistance meter (WPI Company) [32]. After calibration as required, we placed the long end of the electrode in the culture medium outside the cell insert and the short end in the upper culture medium of the cell (taking care not to contact the cell with the wall of the culture plate). The dynamic TEER value was used as the criterion for forming tight cell junctions: $\Omega$ measurement $=\Omega$ actual measurement $\Omega$ blank. The area was used to calibrate TEER: $\Omega / \mathrm{cm} 2=\Omega$ measurement/S, where $S$ is the area of the bottom film of the board [33].

\subsection{BBB permeability test}

Permeability tests with dextran or A labeled with fluorescein isothiocyanate (FITC) $[28,34,35]$ were performed using FITC-dextran (molecular weight $40 \mathrm{kDa}$ ) and FITC-A 1-40 to evaluate the permeability of the in vitro BBB to larger molecules. After the BBB model was established, $100 \mathrm{nM}$ or $1 \mu \mathrm{M}$ AB1-40 was added to the lower chamber of the Transwell container in the experimental group, and then, assay buffer containing $5 \mu \mathrm{M}$ FITC-dextran or FITC-Aß1-40 $(1 \mu \mathrm{M})$ was added. The cells continued to cultivate in the lower chamber of the BBB system. At $0,30,60,90,120$, and $180 \mathrm{~min}$ after addition of fluorescein, $50 \mu \mathrm{l}$ of the medium was taken from the upper chamber and supplemented with an equal volume of medium. $A$ fluorescence microplate reader was used to detect the fluorescence intensity of the sample (excitation wavelength $485 \mathrm{~nm}$; emission wavelength $520 \mathrm{~nm}$ ). According to the standard curve of FITC-dextran/Aß, the relative fluorescence unit was converted to the value of $\mathrm{ng} / \mathrm{ml}$, and the background fluorescence and serial dilution were corrected during the experiment.

\subsection{Aß1-40 preparations}

A 1-40 was mixed to $1 \mathrm{mM}$ with hexafluoroisopropanol, stored in a fume hood to air dry overnight after vortexing to dissolve it, resuspended in DMSO, mixed with $1 \mathrm{mM}$ liquor, and aggregated at $37^{\circ} \mathrm{C}$. This sample was stored at $-80^{\circ} \mathrm{C}$ and diluted with culture medium to the required concentration for use.

\subsection{Gene silencing with lentivirus-mediated siRNA}

LV-CD36-RNAi was obtained from GeneChem. According to the manufacturer's protocols, the lentivirus and negative control sequences were transduced into pericytes. The virus was added according to the appropriate multiplicity of infection (MOI). Puromycin $(2 \mu \mathrm{g} / \mathrm{ml})$ was added after 72 hours to select cells. 
After 1 week of screening, the cells were passaged at 1:2 in T25 bottles, and then, the medium was changed every 2 days. qRT-PCR and immunoblotting experiments were used to detect the silencing efficiency of siRNA, which was then used for the follow-up experiments.

\subsection{Analysis of reactive oxygen species (ROS) production}

After cell treatment, a lipid ROS fluorescent probe (BODIPY 581/591, $50 \mu \mathrm{M}$ ) was added and incubated for 1 hour or intracellular $\mathrm{Fe}^{2+}$ probe (Mito-FerroGreen, $5 \mu \mathrm{M}$ ) working solution was added at $37^{\circ} \mathrm{C}$. After culture for $30 \mathrm{~min}$, the cells were washed 3 times with serum-free medium. Finally, the cells were detected by a multifunctional fluorescence microplate reader.

\subsection{ROS fluorescent probe}

Dihydroethidium (DHE), a superoxide anion fluorescent probe, is bound to DNA by ROS oxide in living cells, and the fluorescence is red. The Reactive Oxygen Species Assay Kit (also called ROS Assay Kit) contains the fluorescent probe DCFH-DA. ROS can oxidize this probe into green-emitting DCF in cells. The probe loading steps were as follows: DHE and DCFH-DA were diluted with serum-free medium to $5 \mu \mathrm{M}$ and $10 \mu \mathrm{M}$, respectively. The cell supernatant was aspirated, the cells were washed twice with PBS, and DHE or DCFH-DA was added to cover the cells. Then, the cells were placed in a $37^{\circ} \mathrm{C}$ cell incubator and incubated for $60 \mathrm{~min}$. The cells were washed twice with PBS before observation, and pictures were taken with a fluorescence microscope to remove the probes in the supernatant.

\subsection{Detection of mitochondrial ROS (MitoSOX) and mitochondrial membrane potential $(\Delta \psi)$}

The $\Delta \psi$ and MitoSOX assays were performed according to the manufacturer's specifications. The cells were treated as described above. After stimulation with A $1-40$, the cells were separated using trypsin and centrifuged at $3000 \mathrm{rpm}$ for $5 \mathrm{~min}$. Then, the cells were resuspended in pericyte culture medium and stained as follows: mitochondrial ROS were incubated with $5 \mu \mathrm{M}$ MitoSOX at $37^{\circ} \mathrm{C}$ for $15 \mathrm{~min}$, and mitochondrial membrane potential was determined with $100 \mathrm{nM} \mathrm{TMRM}$ at $37^{\circ} \mathrm{C}$ for $30 \mathrm{~min}$. After washing, the cells were subsequently measured using an LSR II flow cytometer (BD Biosciences) and analyzed using FlowJo software.

\subsection{Annexin V-FITC/PI double-label staining to detect cell apoptosis}

1. The culture medium was discarded, and the cells were digested with $0.25 \%$ trypsin without EDTA at room temperature. After the cells were round and floating under the microscope, the digestion was 
immediately stopped with complete culture medium, and the cell suspension was transferred to a flow tube.

2. The sample was centrifuged at $1000 \mathrm{rpm}$, and the precipitate was left after $5 \mathrm{~min}$.

3. The cells were washed twice with precooled PBS and resuspended at $1 \times 106 \mathrm{cells} / \mathrm{ml}$ in $1 \times$ buffer.

4. One hundred microliters of the resuspended cell solution was placed in a flow tube.

5. Then, $5 \mu$ of FITC Annexin V and $5 \mu$ l of PI were added.

6. The sample was vortexed gently and incubated for $5 \mathrm{~min}$ at room temperature in the dark.

7. Then, $400 \mu$ of $1 \times$ buffer was added to each tube for testing within 1 hour. The cells were subsequently measured using an LSR II flow cytometer (BD Biosciences) and analyzed using FlowJo software.

The Annexin V-FITC/PI double labeling results were as follows: cells are represented in the four quadrants: lower left (live cells), lower right (early apoptosis) upper right (late apoptosis) and upper left (cell fragments) using FlowJo software Data collection and analysis were performed.

\subsection{Measurement of adenosine-5'-triphosphate (ATP) levels}

ATP levels were determined using an ATP Bioluminescence Assay Kit following the manufacturer's instructions. Briefly, the supernatant was discarded, the lysis solution was added, and the samples were shaken to fully lyse the cells ( $0.2 \mathrm{ml}$ was added to each well of the 6-well plate). After lysis, the cells were transferred to a $1.5 \mathrm{ml}$ EP tube and placed in a $4^{\circ} \mathrm{C}$ centrifuge at $12,000 \mathrm{rpm}$ for $10 \mathrm{~min}$. After washing, the protein concentration was determined by the BCA method. First, $100 \mu \mathrm{l}$ of ATP detection working solution was added to each well of a black 96 -well plate. Then, $20 \mu$ of sample or standard (drawn ATP standard curve) was added and mixed quickly. The samples were immediately tested in multifunctional microplate reader (luminometer) mode. The ATP concentration was converted to $\mu \mathrm{mol} / \mu \mathrm{g}$ according to the protein concentration.

\subsection{Detection of superoxide dismutase (SOD) in the cell supernatant}

The protocol was performed according to the SOD detection kit manual (Nanjing Jiancheng Bioengineering Institute, A001-3). First, after the cells were treated according to the above method, the cell supernatant was transferred to a $1.5 \mathrm{ml}$ EP tube and centrifuged at $2000 \mathrm{rpm}$ for $5 \mathrm{~min}$. After the cells were lysed, the protein concentration was determined by the BCA method, and the samples were used for subsequent experiments. After thorough mixing, the mixture was incubated in a $37^{\circ} \mathrm{C}$ incubator for 20 min and measured at $450 \mathrm{~nm}$ with a microplate reader. Finally, the OD value was converted to $\mathrm{U} / \mathrm{ml}$. 


\subsection{Glutathione peroxidase (GSH-PX) detection}

With a GSH-PX test kit (Nanjing Jiancheng Bioengineering Institute, A005), the enzyme activity can be calculated according to the consumption of reduced glutathione in the enzymatic reaction. Cell protein was extracted according to the above method, and BCA assays were used to measure the protein concentration of GSH-PX. First, the optimal sampling concentration was determined, and an inhibition rate of $45-50 \%$ was taken as the optimal sampling concentration. The inhibition rate formula was as follows: inhibition rate $=$ (nonenzyme tube-enzyme tube) OD value/nonenzyme tube-enzyme tube OD value $\times 100 \%$, and the result was between $15 \%$ and $55 \%$. Then, the OD value of each tube was measured at a wavelength of $412 \mathrm{~nm}$ according to the instructions' steps, and the GSH-PX activity was calculated.

\subsection{Iron content detection}

We used an iron colorimetry kit (Elabscience Biotechnology Co., Ltd.); ferrous iron and pyridine combine to form a pink complex, and the amount of iron ions is proportional to the color. According to the above method, the cell protein was extracted, and BCA was used to measure the protein concentration for quantification of iron ion levels. The protocol was as follows: $\otimes$ The blank tube had $500 \mu$ of deionized water, the standard tube had $500 \mu \mathrm{l}$ of standard application solution with a concentration of $2 \mathrm{mg} / \mathrm{l}$, and the measurement tube had $500 \mu \mathrm{l}$ of the sample to be tested. The samples were separately added to $5 \mathrm{~m}$. EP tubes. $\otimes$ Then, $1.5 \mathrm{ml}$ of iron developer was added to each tube $\otimes$, and the tubes were vortexed, mixed well, and boiled in boiling water at $100^{\circ} \mathrm{C}$ for $5 \mathrm{~min}$. $\otimes$ The samples were cooled with running water and centrifuged for $10 \mathrm{~min}$ at $2300 \times \mathrm{g}$. $\otimes$ Then, $250 \mu \mathrm{l}$ of each supernatant was added to a 96-well plate, and the absorbance was measured at a wavelength of $520 \mathrm{~nm}$. The iron content was calculated according to the following formula: iron content (mg/gprot) $=$ (sample-blank) OD value/(standard-blank) OD value $\times$ standard concentration $(2 \mathrm{mg} / \mathrm{l}) \div$ test sample protein concentration (gprot/l).

\subsection{CCK-8 assay of cell proliferation}

This assay was conducted following the instructions of the Cell Counting Kit-8 (Dojindo, CK04). The steps are as follows: 1 . First, $100 \mu \mathrm{l}$ of cell suspension was prepared in a $96-w e l l$ plate, and the culture plate was placed in an incubator for 24 hours (at $37^{\circ} \mathrm{C}, 5 \% \mathrm{CO}_{2}$ ); 2 . After $\mathrm{A} \beta 1-40$ treatment, the sample was added to the culture plate; 3 . The culture plate was incubated in an incubator for $0,6,12,18$, and 24 hours; 4 . Then, $10 \mu$ of CCK-8 solution was added to each well; 5 . The culture plate was incubated in the incubator for 2 hours; 6 . The absorbance was measured at $450 \mathrm{~nm}$ with a microplate reader.

\subsection{Immunofluorescence and confocal imaging}

After the various treatments, primary cultured mouse cells seeded on coverslips were fixed with $4 \%$ icecold paraformaldehyde at room temperature for $15 \mathrm{~min}$ followed by permeabilization with $0.1 \%$ Triton $\mathrm{X}$ - 
100/PBS. After incubation in blocking solution (2\% BSA/PBS) at room temperature for 1 hour, the cells were incubated with primary antibody at $4^{\circ} \mathrm{C}$ overnight. This step was followed by three washes in PBS. The cells were then incubated with goat anti-rabbit IgG-Alexa 594 (Abcam), goat anti-rabbit IgG-Alexa 488 (Abcam), goat anti-mouse IgG-Alexa 594 (Abcam), goat anti-mouse IgG-Alexa 488 (Abcam), or goat antimouse IgG-Alexa 594 (Arigo) for 1 hour at room temperature. After the cells were washed with PBS 3 times, the coverslips were mounted onto glass slides using Fluorescent Mounting Medium. For visualization of acidic lysosomal compartments, cells were stained with LysoTracker Red (Thermo Fisher Scientific) for 1 hour according to the manufacturer's instructions. After incubation with LysoTracker Red, cells were fixed in $4 \%$ PFA/PBS for $15 \mathrm{~min}$ at room temperature. The cells were incubated with primary antibody at $4^{\circ} \mathrm{C}$ overnight. The cells were then washed in PBS 3 times followed by staining with $2 \mathrm{mg} / \mathrm{ml}$ Hoechst 33342 for 5 min at room temperature. The cells were washed with PBS four times, and coverslips were mounted on glass slides using Fluorescent Mounting Medium. All confocal images were acquired and processed using a confocal microscope (Nikon).

\subsection{Western blotting}

Proteins in the animal brain were homogenized, and cells were lysed with RIPA buffer. Samples were loaded on SDS-PAGE (8-12\% acrylamide) gels. The total protein concentration was determined by BCA assays. The same amount of protein (20-40 mg) was loaded and run on SDS-PAGE. The proteins were separated by electrophoresis and transferred to nitrocellulose membranes $(0.45$ or $0.22 \mu \mathrm{m})$. The membranes were blocked with 5\% nonfat dry milk in $0.01 \mathrm{M} \mathrm{PBS} \mathrm{(pH} \mathrm{7.4)} \mathrm{and} 0.05 \%$ Tween-20 (PBST) at room temperature for 1 hour. Subsequently, the membrane was incubated with primary antibodies directed against target proteins overnight at $4^{\circ} \mathrm{C}$. The final dilutions for primary antibodies were as follows: after three quick washes in PBST, the membranes were incubated with secondary antibodies conjugated to horseradish peroxidase (Abcam) diluted at 1:3,000 in PBST for 1 hour. Final detection of immunoreactive bands was developed using an enhanced chemiluminescent Western blot system (ECL) with exposure to a chemiluminescence imaging system (UVP). Immunoblotting signal intensity was measured using ImageJ 64 software.

\subsection{Electron microscopy}

For scanning electron microscopy, the cells were grown and processed as described above. First, the cells were fixed with $2.5 \%$ glutaraldehyde at $4^{\circ} \mathrm{C}$ overnight and then washed three times with PBS. After being fixed with $1 \%$ osmium acid for 1.5 hours at $4^{\circ} \mathrm{C}$, the samples were dehydrated in a graded ethanol series and embedded in acrylic resin at $60^{\circ} \mathrm{C}$ for 48 hours. Then, 70-nanometer ultrathin sections were mounted on a nickel grid. The samples were stained with uranyl acetate for 10-20 min and lead citrate for 5-10 min and rinsed thoroughly with distilled water. A $120 \mathrm{kV}$ Hitachi transmission electron microscope was used to take pictures of the sample. 


\subsection{Quantitative real-time PCR and sequencing.}

Total RNA was extracted from cells using TRIzol (Invitrogen) and further washed using RNase-free water. RNA was reverse-transcribed with a PrimeScript RT Reagent kit (TaKaRa) for CDNA synthesis, and the cDNA was then amplified by real-time PCR with a SYBR Premix ExTaq kit (TaKaRa). The relative expression of genes was normalized to the expression of the housekeeping gene GAPDH. Primer sequences were as follows: GAPDH: forward: 5'-GTTCCTACCCCCAA TGTGTCC-3', reverse: 5'TAGCCCAAGA TGCCCTTCAGT-3'; CD36: forward: 5'- ACCTTTTGTTGAGAAGTCTCGAAC-3', reverse: 5'CTTTTTCAGTGCAGAAACAGTGG-3'; LRP1: forward: 5'- CTGGTCGATAGCAAGATTGTATTTC-3', reverse: 5'GTGGCGTAGAGGTAGTTCTCAAAC -3'; HSP60: forward: 5'- CAGTCCTTCGCCAGATGAGAC -3', reverse: 5'GGGACTTCCCCAACTCTGTTC -3'; TIM23: forward: 5'- ACTGGTATGAACCCCCTGTCTC -3', reverse: 5'CTGAGTTTCCTTCAATCCTAAACG -3'. Analysis was performed with three biological replicates and three independent technical replicates for each sample, with the average value taken and normalized to GAPDH gene levels to give the threshold cycle $(\Delta \mathrm{CT})$ values.

\section{Statistical Analysis}

All data are expressed as the mean \pm standard deviation, and SPSS 23.0 software (SPSS, Inc., Chicago, Illinois, USA) was used for analysis of significant differences. GraphPad Prism 8.0 software was used for drawing. T-tests were used for comparison between the two groups. One-way analysis of variance was used to compare multiple groups, and Student-Newman-Keuls (SNK) tests were used for comparison between groups. For repeated measurement data, repeated measurement analysis of variance was used. For comparison of data between two groups, Student's t test was used when the variance was uniform, and Welch's $t$ test was used when the variance was not uniform. $p<0.05$ indicated significant differences. If not indicated, the $p$ values were not significant $(>0.05)$.

\section{Result}

\subsection{The BBB of APP/PS1 mice is destroyed, the number of pericytes decreases, and $A \beta$ is phagocytosed through CD36}

Normal glucose metabolism in brain tissue requires a complete BBB. Under normal circumstances, the glucose uptake of brain tissue is maintained at a high level. As neuronal apoptosis and destruction of the BBB increase in APP/PS1 mice, glucose uptake in the brain tissue begins to decrease. We first observed 18F-FDG uptake in brain tissue with PET/CT. The blood perfusion imaging of 9-month-old and 6-monthold APP/PS1 mice showed an asymmetrical reduction in frontal and temporal lobe perfusion. We found that $18 \mathrm{~F}-\mathrm{FDG}$ uptake is reduced (Figure 1A).

The immunoblotting results of the total proteins in the cerebral cortex showed that the expression levels of the pericyte-specific proteins PDGFR $\beta$ and NG2 in the APP/PS1 mice gradually decreased. The levels were significantly different compared with those of the WT group. The expression level of CD36 was 
most significantly lower in the 9-month-old APP/PS1 mice than in the WT mice. In contrast, the expression level of the A $\beta$ transporter LRP1 was not substantially different from that of the WT group (Figure 1B).

Previous studies have confirmed that the lack of CD36 can offset cerebrovascular dysfunction in Tg2576 mice and partially normalize the number and morphology of pericytes [25]. In this study, we confirmed by immunohistochemistry that 6-month-old APP/PS1 mice had A $\beta$ plaques (Figure 1C), and the pericyte numbers decreased (Figure 1D). To determine whether pericytes phagocytose $A \beta$ through $C D 36$, we performed immunofluorescence costaining for CD36, A $\beta$, and PDGFR $\beta$ (labeled pericytes) on APP/PS1 mouse brain slices to detect whether the three proteins colocalized, and we found (Figure 1E) that pericytes can engulf $A \beta$ through CD36. However, the specific mechanism that affects the BBB is unknown. Therefore, we designed an in vitro BBB model to further verify the role of pericyte CD36 in the BBB.

\subsection{Pericytes phagocytose $A \beta 1-40$ via CD36, causing injury to the BBB in vivo}

\subsubsection{Pericytes engulf $A \beta 1-40$ by CD36}

To further verify whether the pericytes enter the cells through CD36 phagocytosis of A 1 1-40, we used HiLyte FluorTM 555-AB1-40 with red light. Then, immunofluorescence staining for CD36 and light microscopy showed pericytes under white light, and red fluorescent A 1-40 overlapped (Figure 2A), suggesting that $A \beta 1-40$ is transported into the cell. Furthermore, we found that CD36 (green) under immunofluorescence and $A \beta 1-40$ with red fluorescence colocalized in pericytes (Figure 2B), confirming that pericytes can engulf $A \beta 1-40$ via CD36.

\subsubsection{A low dose of $A \beta 1-40$ leads to upregulation of CD36 expression in pericytes, while the transporter LRP1 is not affected}

To verify the effect of $A \beta 1-40$ on the expression of pericyte transporters, we used two doses of $A \beta 1-40$ $(100 \mathrm{nM}$ and $1 \mu \mathrm{M})$ to verify the cell level. After treatment with $100 \mathrm{nM}$ and $1 \mu \mathrm{M} A \beta 1-40$ and incubation with pericytes for 6 hours, both the protein level (Western blot) (Figure 2C) and the transcription level (PCR) (Figure 2D) indicated that the expression of CD36 was significantly increased after stimulation with $A \beta 1-40$. In addition, the concentration of $1 \mu \mathrm{M} A \beta 1-40$ increased more significantly $(p<0.001)$. At this time, the protein level (Figure $2 \mathrm{C}$ ) and the transcription level (Figure 2E) of the amyloid efflux transporter LRP1 did not change significantly. To further verify the function of CD36 in the BBB, we performed RNA interference to downregulate the expression of CD36 at the gene level and transferred the si-CD36 plasmid via lentivirus to alter expression at the protein level (Figure 2F) and transcription level (Figure $2 \mathrm{G})$. We found that the CD36 expression level after RNA interference was significantly lower than that of the control group, confirming the efficacy of the interference. Moreover, there was no difference compared 
the empty vector control group (si-NC group). After downregulating the expression of CD36, we performed follow-up studies of the BBB in vitro.

4.2.3 $A B 1-40$ increases the permeability of the $B B B$, and downregulating the expression of CD36 in pericytes improves the permeability changes in the BBB induced by $A \beta 1-40$

Because AB1-40 is easily deposited in the vascular system [36], its impact on the endothelial cells that constitute brain microvessels and the pericytes that share the basement membrane with endothelial cells and help regulate capillary diameter and cerebral blood flow leads to changes in the BBB. The choice of clinical treatment targets is pivotal. To further verify the effect of $A \beta 1-40$ on the permeability of the BBB, we cultured bEnd. 3 cells separately and cocultured primary pericytes and bEnd. 3 cells in an in vitro Transwell system to establish tight junction-type in vitro BBB model.

Regarding the effect of CD36 pericytes on the permeability of the BBB, we inhibited the expression of CD36 by lentiviral transfection through RNA interference and then cocultured primary pericytes and bEnd. 3 cells in the Transwell system to establish a contact-type in vitro BBB model. Endothelial cells were seeded on the front side of the Transwell cell membrane, and pericytes were seeded on the backside of the cell membrane. The two cell types were in contact through the intermembrane pores (pore size 0.4 $\mu \mathrm{m}$ ). This model can ideally simulate the in vivo BBB structure (Figure $3 \mathrm{C}$, simulation diagram in D).

In this experiment, after construction of an in vitro BBB model, $A \beta 1-40$ (100 nM and $1 \mu \mathrm{M})$ was first added to the lower chamber of a Transwell plate in a single culture and coculture model for 6 hours, and then, the medium was changed and the fast connectivity of the BBB was tested by TEER. The change reflects the influence of $A \beta 1-40$ on the BBB. We found that the TEER in the coculture model was significantly higher than that in the bEnd.3 single-culture models (Figure 3A). The difference was significant. A 1 1-40 (1 $\mu \mathrm{M}$ ) dramatically reduced the TEER value of BBB cells cultured alone and cocultured (Figure $3 A$ ). The difference was significant $(p<0.05)$, while $100 \mathrm{nM} A \beta 1-40$ had no effect on tight junctions in the BBB cells cultured alone and cocultured (Figure $3 A$ ). These results indicate that pericytes can increase the tightness of the BBB and that $A \beta 1-40$ damages the BBB in a dose-dependent manner.

After inhibition of CD36 expression in pericytes, the TEER value of the BEC/PC si-CD36 group in the cocultured Transwell model was significantly higher than that of the control group $(p<0.05)$ (Figure 3B). However, the TEER value of the $1 \mu \mathrm{M}$ Aß1-40-treated group was significantly decreased compared with that of the control group 6 hours after AB1-40 was added to the lower chamber, while the value in the $\mathrm{BEC} / \mathrm{PC}$ si-CD36 group was also reduced. Nevertheless, there was no significant difference compared to the control group. Permeability testing of FITC-labeled A 1 1-40 and dextran (dextran, $40 \mathrm{kDa}$ ) was performed; $1 \mu \mathrm{M}$ AB1-40 was added to the lower chamber 6 hours after the BBB permeability increased significantly, and after inhibiting CD36, the labeled $A \beta 1-40$ and the penetration of dextran in the BEC/PC si-CD36 group were markedly lower than those in the BEC/PC group (Figure $3 C, D$ ). The above results 
indicate that $1 \mu \mathrm{m} \mathrm{A \beta 1-40} \mathrm{increases} \mathrm{the} \mathrm{permeability} \mathrm{of} \mathrm{the} \mathrm{BBB,} \mathrm{and} \mathrm{inhibiting} \mathrm{the} \mathrm{expression} \mathrm{of} \mathrm{CD36} \mathrm{in}$ pericytes can reduce the permeability of the BBB and increase its compactness.

\subsection{A low dose of $A \beta 1-40$ inhibits pericyte proliferation, causes mitochondrial damage, and promotes mitophagy}

To study the effect and influence of low-dose $A \beta 1-40$ on pericytes, we incubated pericytes with different concentrations ( $100 \mathrm{nM}$ and $1 \mu \mathrm{M})$ of $A \beta 1-40$ for 6 hours to observe the impact on pericyte proliferation and mitochondria. In this study, a CCK-8 kit was used to detect the effect of A 1-40 on pericyte proliferation. The results showed that the proliferation of pericytes was significantly inhibited after A 31 40 treatment for 12 hours (Figure $4 \mathrm{~A}$ ), and $1 \mu \mathrm{M}$ resulted in the maximum inhibition at 24 hours The results were significant $(p<0.01)$, indicating that a low dose of $A \beta 1-40$ can inhibit the proliferation of pericytes in a time- and concentration-dependent manner.

We also used flow cytometry to observe the effects of $A \beta 1-40$ stimulation on pericyte mitochondrial damage and found that $A \beta$ caused a significant increase in mitochondrial ROS (MitoSOX) compared with that of the control group (Figure 4B). In contrast, the mitochondrial membrane potential (TMRM) was significantly higher than that of the control group (Figure $4 \mathrm{C}$ ), with $1 \mu \mathrm{M}$ showing the most significant effect $(p<0.01)$. This finding indicates that with increasing $A \beta 1-40$ concentration, the damage to pericyte mitochondria is more serious and is concentration-dependent.

To further explore the effect on mitochondria, we tested the mitochondrial matrix protein HSP60 (Figure 4D) and the inner membrane protein Tim23 (Figure 4D). The results showed that the expression of the two mitochondrial proteins was significantly reduced after $A \beta 1-40$ treatment $(p<0.01)$, and the expression of LC3II/LC3I (Figure 4D) showed a significant increase $(p<0.01)$ after autophagic activation, indicating that $A \beta 1-40$ treatment of pericytes caused an increase in mitochondrial autophagy in pericytes and promoted autophagy and the formation of autophagic bodies. We found that $1 \mu \mathrm{M} \mathrm{A \beta 1-40} \mathrm{showed} \mathrm{a}$

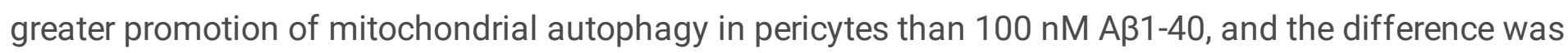
significant $(p<0.05)$. We also examined the expression of the mitochondrial matrix protein HSP60 (Figure 4E) and the inner membrane protein Tim23 (Figure 4F) at the RNA level after A 1-40 treatment. The findings were consistent with the decrease in protein levels caused by A $1-40$, but the values were between those induced by the two doses. The difference was not significant.

To further verify the effect of A 1-40 on pericyte mitochondrial autophagy, we also used immunofluorescence to detect changes in autophagic flux. We the examined mitochondrial matrix protein HSP60 and autophagosomes (LC3) after treatment with $1 \mu \mathrm{M} \mathrm{A \beta 1-40} \mathrm{and} \mathrm{observed} \mathrm{colocalization} \mathrm{of} \mathrm{the}$ proteins (Figure 4G). To confirm that lysosomes indeed degraded mitochondria, we used a lysosomal tracer to costain mitochondria and lysosomes and found that $1 \mu \mathrm{M} \mathrm{A \beta 1-40} \mathrm{stimulated} \mathrm{mitochondria} \mathrm{and}$ lysosomes. The colocalization of the enzyme body (LysoTracker Red) was significantly increased (Figure $4 \mathrm{H})$, indicating that autophagic flux increased after stimulation with $A \beta 1-40$; thus, $A \beta 1-40$ induced 
lysosome-mediated mitochondrial degradation. However, there was no significant overlap between the control group and the DMSO group. Our results suggest that A $\beta 1-40$ stimulates pericytes to increase mitochondrial autophagy, which involves mitochondria and fusion with lysosomes for degradation.

To further confirm that Aß1-40 promotes pericyte mitochondrial autophagy, we applied the autophagic agonist carbonyl cyanide 3-chlorophenylhydrazone (CCCP; a respiratory chain uncoupler that reduces the mitochondrial membrane potential and induces mitochondrial autophagy; $5 \mu \mathrm{M}$ ) to treat pericytes for 6 hours. The results showed that the levels of the mitochondrial matrix protein HSP60 (Figure 5A) and the inner membrane protein Tim23 (Figure 5A) were significantly lower than those of the control group. In contrast, the expression of LC3II/LC3I (Figure 5A) after autophagic activation showed a significant increase, consistent with the results of $A \beta 1-40$ at $1 \mu \mathrm{M}$. We also used the autophagy inhibitor chloroquine (CQ, which inhibits autophagosome and lysosome fusion by inhibiting autophagy; $20 \mu \mathrm{M}$ ) and added CQ 2 hours before adding AB1-40. We found that after adding CQ, AB1-40 induced HSP60 (Figure 5B) and Tim23 expression (Figure $5 B$ ). The decrease was reversed, and the levels of the two mitochondrial proteins were significantly higher than those in the $A \beta 1-40$ group $(p<0.01)$. Electron microscopy data (5D) showed that after pericytes were stimulated by $A \beta 1-40$ or treated with $C C C P$, mitochondria were surrounded by autophagosomes/autolysosomes. The above results further confirmed that AB1-40 induces an increase in mitochondrial autophagy in pericytes.

We also use the drug Mdivi-1 for verification. Mdivi-1 is an inhibitor of mitochondrial division and is often used to inhibit mitochondrial autophagy [37, 38]. After adding Mdivi-1 (20 $\mu \mathrm{M}) 2$ hours before adding $A \beta 1-40$, we found that the reduction in HSP60 and Tim23 expression induced by A $1-40$ was blocked by Mdivi-1 (Figure $5 \mathrm{C}$ ), and compared with the $A \beta 1-40$ group, the treated group showed a significant difference $(p<0.01)$. After Mdivi-1 treatment of pericytes, the electron microscopy results (Figure 5D) showed that the area surrounding the pericyte mitochondria with autophagosomes/autolysates was better than that in the AB1-40 treatment group, and the number of mitochondria increased. The results showed that Mdivi-1 could inhibit mitochondrial autophagy caused by Aß1-40. We verified that $1 \mu \mathrm{M} \mathrm{A \beta 1-}$ 40 induces an increase in mitochondrial autophagy in pericytes.

\subsection{Low-dose $A \beta 1-40$ does not cause pericyte cell apoptosis, but oxidative stress increases}

Mitochondrial damage and increased mitochondrial autophagy often lead to apoptosis. To further verify whether A 1 1-40 (100 nM and $1 \mu \mathrm{M})$ causes pericyte apoptosis, we detected changes in apoptosis by flow cytometry and observed apoptosis at the protein level. We examined changes in proteins such as the antiapoptotic protein BCL2, the proapoptotic protein BAX, and the key apoptotic proteins caspase 3 (P35) and cleaved-caspase 3 (P17). We found that BCL2/BAX (Figure 5E) and P17/P35 CASP3 (Figure 5E) levels did not change significantly after AB1-40-stimulation of pericytes compared with that of the control group. The apoptosis rate was detected by flow cytometry with Annexin-V/PI double staining to determine the changes in apoptosis. The results showed no difference (Figure 5F). The above results indicate that a low dose of AB1-40 did not cause apoptosis within 6 hours, but we found that pericyte 
mitochondria were damaged (Figure 4). The BBB was destroyed (Figure 3). To further clarify the cause of cell damage, we detected oxidative stress.

ROS fluorescent probes (DHE and ROS kits) can freely penetrate the living cell membrane, can enter the cell and are oxidized by ROS in the cell to produce red and green fluorescence. Our study found that with $1 \mu \mathrm{m}$ A 1 1-40, DHE red fluorescence (Figure 5G) and ROS green fluorescence (Figure 5H) were significantly enhanced, leading to an increase in pericytic ROS, and the production of ATP (Figure 5I) was considerably lower than that of the control group. During this process, SOD (Figure 5J) showed no significant change. The above results indicate that A $\mathrm{B} 1-40$ leads to an increase in cellular ROS production and a decrease in energy synthesis.

\subsection{A low dose of $A \beta 1-40$ induces ferroptosis in pericytes}

\subsubsection{A low dose of $A \beta 1-40$ induces an increase in iron ions and lipid ROS in pericytes, leading to increased ferroptosis}

Ferroptosis is a new form of cell necrosis. The primary mechanism is the accumulation of cellular lipid ROS in an iron-dependent manner [39]. The cystine/glutamate transporter SLC7A11 (also known as xCT) imports cysteine for glutathione biosynthesis and antioxidant defense [40]. Glutathione peroxidase 4 (GPX4) is an enzyme required to remove lipid ROS. Even under normal cellular cysteine and glutathione contents, this enzyme can induce iron-related death and is crucial for ferroptosis [41]. We found that stimulating cells with a low dose of A 1-40 increased ROS production in pericytes and damaged mitochondria. Therefore, we suspected that AB1-40 might cause ferroptosis in pericytes. To verify the above assumptions, we treated cells with $1 \mu \mathrm{M} A \beta 1-40$ for one week. The cells were tested for related iron death indicators after 6 hours, and erastin, an inducer of iron death, was used as a positive control.

We found that $A \beta 1-40$ caused a significant decrease in GSH-Px expression compared with that of the control group (Figure 6A). The level of iron ions increased significantly compared with that of the control group (Figure 6B), and the trend was the same as that after erastin stimulation. The results showed that both erastin and $A \beta 1-40$ treatment of pericytes resulted in a significant increase in lipid ROS (Figure 6C) and $\mathrm{Fe}^{2+}$ (Figure 6D) compared to those of the control group. After treatment of pericytes with erastin and $\mathrm{A} \beta 1-40$, the expression levels of the proteins GPx4, $\mathrm{xCT}$, and ferritin (Figure 6E) were significantly lower than those in the control group, and the expression level of NOX1 (Figure 6E) was slightly increased compared with that in the control group. However, the difference was not significant. The above results show that $A \beta 1-40$ induces ferroptosis of pericytes.

\subsubsection{Low-dose $A \beta 1-40$ causes ferroptosis reliant on mitochondrial autophagy in pericytes}

We also applied ferrostatin-1 (FER-1), a ferroptosis inhibitor, to confirm that A $\beta 1-40$ induces ferroptosis in pericytes. Previous studies have shown that ferroptosis is an autophagic cell death process [42], so we 
used Mdivi-1 for verification. Through Western blotting, we found that after adding $10 \mu \mathrm{M}$ FER-1, the reduction in the iron death-related proteins GPx4, ferritin, and XCT induced by A 1-40 was blocked by FER1 (Figure 6F). The difference was significant compared with the A $\beta 1-40$ group ( $p<0.05$ or 0.01 ). After $A \beta 1$ 40 or erastin treatment of pericytes, transmission electron microscopy (Figure $6 \mathrm{H}$ ) showed mitochondrial atrophy, increased membrane density, and necrosis-related vacuoles, which are morphological features of iron death [43], and FER-1 reversed the mitochondrial changes associated with iron death. The above results indicate that FER-1 can inhibit mitochondrial autophagy caused by A $1-40$.

The results showed that after addition of Mdivi-1, the reduction in GPx4 and ferritin levels induced by A 1-40 could be reversed by Mdivi-1 (Figure 6G), and the difference was significant compared with the A 1-40 group $(p<0.01)$. However, $x C T$ did not change significantly after Mdivi-1 treatment of pericytes compared with that of the A 1-40 group (Figure 6G). These results show that Mdivi-1 inhibits ferroptosis by inhibiting cell mitochondrial autophagy and then upregulating the expression of GPx4. The above results indicate that the ferroptosis induced by AB1-40 is mediated by mitochondrial autophagy.

\subsubsection{Low-dose A $1-40$ induces mitochondrial autophagy in pericytes through the CD36/PINK1/Parkin pathway}

To further explore the mechanism by which $A \beta 1-40$ induces mitochondrial autophagy, we first detected the levels of the mitochondrial pathway proteins S65, BNIP3/NIX, and PINK1/Parkin in pericytes by Western blotting. We found that only PINK1/Parkin pathway proteins increased significantly after $1 \mu \mathrm{M}$ A $31-40$ treatment of pericytes (Figure 6I). Compared with the control group, the difference was significant $(p<0.01)$. S65 and $1 \mu \mathrm{M}$ BNIP3/NIX Aß1-40 treatment decreased the protein level of pericytes (Figure 6I). The results showed that $A \beta 1-40$ induced pericytes to activate mitochondrial autophagy through the PINK1/Parkin pathway.

To study the effect of CD36 on changes in the mitochondrial autophagic pathway, we downregulated the expression of CD36 in pericytes and then treated them with $1 \mu \mathrm{M} A \beta 1-40$. The results showed that the expression of CD36 after si-CD36 treatment was significantly lower than that of the control group (Figure $6 \mathrm{~J})$. Western blot results showed that si-CD36 can block the expression of the pericyte mitochondrial pathway protein PINK1/Parkin (Figure $6 \mathrm{~J}$ ) induced by AB1-40 $1 \mu \mathrm{M}$, indicating that si-CD36 blocks PINK1 caused by A $1-40 /$ Parkin pathway activation. Moreover, si-CD36 reversed the downregulation of the A $A 1-$ 40-induced mitochondrial protein HSP60 and Tim23 levels (Figure 6J), indicating that si-CD36 can inhibit $A \beta 1-40$-induced mitochondrial autophagy. The above results suggest that the promotion of mitochondrial autophagy by $A \beta 1-40$ is mediated through the CD36/PINK1/Parkin pathway.

\section{Discussion}

$A \beta$ plays an essential role in $A D$ [44]. To date, many studies have focused on explaining the transcellular movement of A $\beta$ through LRP1 and RAGE receptors $[32,45,46]$. Studies have found that CD36 deletion improves neurovascular function, increases the number of pericytes, and improves pericyte morphology in Tg2576 mice [25]. Mice at 18-22 months of age are characterized by extensive amyloid deposits in 
cerebral blood vessels $[47,48]$. The lack of CD36 in Tg2576 mice aged 3-4 months did not change the amyloid $\beta$ levels in the brain [49]. Therefore, previous studies did not determine the role of CD36 expression on pericytes in the BBB of APP/PS1 mice at 6-9 months. Thus, in this study, APP/PS1 mice aged 6 to 9 months were selected. First, the metabolic rate of brain glucose was gradually decreased, as shown by PET/CT. The levels in the 9-month-old APP/PS1 mice decreased more significantly. At 6 months of age, BBB destruction and neurological dysfunction could already be detected. Consistent with the previous results of reduced glucose metabolism detected by $18 \mathrm{~F}-\mathrm{FDG}$ positron emission tomography with AD-related encephalopathy [50,51], these abnormal changes are most prominent in the fragile hippocampal structure and cortical areas. We also found that the decline in its metabolic rate was asymmetric, which may be related to the distribution and metabolic characteristics of $A \beta$ in the brain. The destruction of the BBB will cause a series of vascular and nerve reactions, such as BBB dysfunction, neuronal damage, neurodegeneration, and potential pathogenic links between neurological disorders.

Moreover, the relationship between neurovascular integrity, brain structure and functional connectivity, cognitive function, and neurosymptoms such as complex AD still needs to be directly explored in the most relevant in vivo environment. Western blot analysis of the whole-brain protein sample showed that the expression of CD36 was reduced when the expression of A 3 mediated through LRP1 is normal, which may indicate that CD36 was activated or inhibited in the APP/PS1 mice while LRP1 was not affected. CD36 is mainly expressed in macrophages and microglial cells, and CD36, as a receptor for microglial inflammation, has been confirmed in AD mouse models [23]. For the decrease in CD36 protein expression in the whole brain, whether its relationship with pericytes will affect the BBB provides a new direction for future research.

Our study found that the brain pericytes of APP/PS1 mice at the age of 6 months were reduced earlier than those of 18- to 22-month-old Tg2576 mice in the literature [25], suggesting that $A \beta$ damage to pericytes may appear in AD. Early targeting and regulation of pericytes may represent a new therapeutic strategy for $A D$ treatment in the early stage. AD patients $[9,52]$ and APP mice $[25,53]$ showed pericyte degeneration and loss. This finding is consistent with the previous finding that excess $A \beta$ in pericytes can induce cell death in human [54] and mouse [53] pericytes. Excessive A $\beta$ deposition in pericytes often causes pericytes to die during this accumulation, and pericyte death will aggravate the pathological progress of $A D$. Recent stem cell studies have shown that when mouse mesoderm pericytes are injected into the brains of APP mice, they can improve the cerebral blood flow of mice and reduce the production of $A \beta$ pathology [55]. Furthermore, pericytes play a decisive role in maintaining the integrity and compactness of the BBB.

This study found that 6-month-old APP/PS1 mice had A $\beta$ plaques formed and deposited in the blood vessels, while 3-month-old Tg2576 mice showed no changes in the structure of cerebral blood vessels, and there was no amyloid sedimentation in the brain or blood vessels [56]. The accumulation of $A \beta$ indicates that the $A \beta$ balance in the body has been disrupted. Vascular transport is the fundamental way to clear $A \beta$ from the brain [57] and the tight junctions of the endothelium at the BBB prevent $A \beta$ from freely entering the blood, so they must pass through a special transporter in the endothelium [19]. In the 
early and late stages of $A D$, the clearance of $\beta$-amyloid appears to be impaired. Fluorescence results showed that PDGFR $\beta, A \beta$, and CD36 are colocalized in the brain, indicating that pericytes can phagocytose $A \beta$ through $C D 36$.

Moreover, the damage to pericytes itself due to the removal of $A \beta$ may also lead to the further development of capillary CAA $[58,59]$. Especially in the APP mouse model, clearance mechanisms such as the perivascular pathway and/or meningeal lymphatic $\beta$-amyloid can no longer meet the needs of the body $[10,11]$. However, the response of $C D 36$ on pericytes to $A \beta$ and whether it affects the BBB requires further research. We also explored whether CD36 is a potential therapeutic target to control the clearance and level of $A B$ in $A D$. To solve this problem, we established a $B B B$ model in vitro to further study the role and mechanism of pericyte CD36 in a BBB coculture model with AB1-40.

Because pericytes are also highly susceptible to cellular stress from various endogenous and exogenous toxins $[7,60]$ (including $A \beta$ ), pericyte death may occur after intracellular accumulation [53]. Therefore, in an in vitro study, we used low doses $(100 \mathrm{nM}$ and $1 \mu \mathrm{M})$ of Aß1-40 to stimulate primary pericytes for 6 hours and then conducted experiments. The study showed that the expression level of CD36 was significantly higher than that of the control group. Previous studies have found that monocytes, microglia, platelets, and endothelial cells highly express CD36 [22, 61, 62], but few reports have described CD36 expression in pericytes. Our results indicate that CD36, as a phagocytic receptor, exists in pericytes. In this study, a pericyte and endothelial cell coculture was used to construct a BBB model. The TEER value was significantly higher than that of the endothelial cell culture model alone, indicating that pericytes play a pivotal role in the formation of the BBB.

Our research found that AB1-40 can aggravate BBB damage by binding to CD36 on the surface of pericytes, resulting in decreased TEER of the BBB and increased permeability after treatment with a low dose of $A \beta 1-40$ in a short time. In addition, this treatment can lead to the destruction of the BBB. Gene knockdown of CD36 expression alleviated the increase in BBB permeability and the destruction of compactness induced by A $31-40$. This finding is consistent with the concept of restoration of microvascular function in APP mice lacking CD36 [25]. The collapse of the BBB in the early stages of the disease is often related to pericytes $[27,53]$. Our results are similar to the above findings, indicating that A 1-40 destroys the integrity of the BBB through pericyte CD36 molecules. These findings confirm the previously unrecognized role of CD36 in the mechanism of vascular amyloid deposition, revealing that CD36 may be a potential therapeutic target for the BBB.

The degradation of $A \beta$ cells is usually carried out through three pathways: the ubiquitin-proteasome pathway, autophagy-lysosomal pathway, and endosome-lysosomal pathway, or it is transported outside of the cell by exocytosis [63]. Our study found that A $31-40$ inhibited pericyte proliferation in a time- and dose-dependent manner. AB1-40 $(1 \mu \mathrm{M})$ caused a significant increase in mitochondrial ROS and decreased membrane potential, indicating that mitochondria were damaged. Damaged or redundant mitochondria are often degraded, and mitochondrial components can be recovered through mitochondrial autophagy $[64,65]$. The expression of the mitochondrial inner membrane protein Tim23 
and the matrix protein HSP60 decreased significantly after stimulation with $1 \mu \mathrm{M} A \beta 1-40$, while the ratio of LC3II/LC3I increased significantly, indicating that A $\beta$ induced an increase in mitochondrial autophagy $[66,67]$. These results were further confirmed by the autophagy agonist CCCP and inhibitor CQ combined with the mitochondrial inhibitor Mdivi-1, which showed that $A \beta$ induced pericyte mitochondrial autophagy. Changes in the autophagic process play an essential role in the pathogenesis of many neurodegenerative diseases. Usually, $A \beta$ is degraded in lysosomes due to high levels of autophagy. Nevertheless, in disease conditions, $A \beta$ accumulates in many autophagosomes in dystrophic neurites and becomes the main intracellular reservoir of toxic peptides in the AD brain [68].

In $A D$, the accumulation of $A \beta$ leads to degeneration of the lysosomal membrane, loss of pyramidal neurons, neuronal cell death, and further neuronal autophagic degradation, which ultimately leads to neurodegeneration $[69,70]$. Our results showed that $A \beta 1-40$ causes BBB destruction after stimulation of pericytes. To explore the mechanism of action, we tested the effect of AB1-40 on pericytes at the level of apoptosis. However, $1 \mu \mathrm{M}$ Aß1-40 did not cause apoptosis of pericytes. Our findings are consistent with the results of a previous study [71], except that they used fibrinogen to stimulate pericytes, which initially activated autophagy but did not activate caspase 3 and/or kill pericytes in the early stage. In recent years, increasing data have demonstrated the role of mitochondria in the pathogenesis of neurodegenerative diseases and apoptosis [72]. Mitochondria are considered the primary source of ROS, and excessive accumulation can cause oxidative damage $[73,74]$. Damaged mitochondria increase ROS production, and excessive ROS cause more damage to mitochondria [75, 76]. We found that A $\beta 1-40$ increased ROS and decreased ATP production in pericytes, while SOD, which has antioxidant effects, did not increase.

ROS accumulate in an iron-dependent manner, leading to a new form of programmed necrosis called ferroptosis [39]. Ferroptosis is related to many diseases, including neurodegenerative diseases, acute renal failure, and cancer cardiomyopathy $[77,78]$. We found that $A \beta 1-40$ caused an increase in $\mathrm{Fe}^{2+}$ in pericytes, increased lipid ROS, and decreased GSH-Px. We further confirmed that A 1 1-40-induced ferroptosis occurs in pericytes by inhibiting GPx4 and xCT. GPx4 is a glutathione-dependent antioxidant enzyme that reduces membrane phospholipid hydroperoxide to inhibit ferroptosis $[41,78,79]$. The function of XCT is to import cysteine for glutathione biosynthesis and antioxidant defense [40].

Ferroptosis often leads to an abnormal increase in iron. Ferritin is an iron storage protein complex used to store excess iron. Many ROS-producing enzymes, such as nicotinamide adenine dinucleotide phosphateoxidase (NOX), lipoxygenase, xanthine oxidase, and cytochrome P450 enzymes, contain iron in their active centers $[43,80]$. In this experiment, $A \beta 1-40$ stimulated pericytes, and no significant increase in NOX1 was found, indicating that NOX did not release $\mathrm{Fe}^{2+}$. We also found that ferroptosis was blocked by Mdivi-1, suggesting that A 1-40 causes autophagy-dependent ferroptosis.

We found that AB1-40 activates pericyte mitochondrial autophagy through the PINK1/Parkin pathway induced by PTEN. PINK1 is a mitochondrial-targeted serine/threonine kinase, and Parkin is a cytoplasmic ubiquitin E3 ligase. When mitochondria are damaged, the loss of mitochondrial membrane potential will result in PINK 1 accumulation on the outer mitochondrial membrane (OMM) [81]. PINK1 mediates the phosphorylation of ubiquitin serine 65 (Ser65) and equivalent serine residues in the ubiquitin-like domain 
of Parkin, leading to Parkin activation and recruitment [81-83]. Activated Parkin generates ubiquitin chains on OMM proteins [82] to interact with the autophagosome resident protein LC3 [84, 85]. Then, they merge and fuse into a complete ring, separating each damaged mitochondria into a mitochondrial autophagosome [26]. After the mitochondria are engulfed, the mitochondrial phagosome fuses with the lysosome, leading to the degradation of damaged mitochondria $[82,86]$. The OMM proteins NIP3-like protein X (NIP3-like protein X, NIX, also known as BNIP3L) and BCL2 interacting protein 3 (BNIP3) are related to mitochondrial morphology and mitochondrial autophagy $[87,88]$. These proteins were initially identified as proapoptotic proteins [89]. Under hypoxic conditions, BNIP3 and NIX are also transcriptionally regulated by hypoxia-inducible factor-1 (HIF-1) and participate in mitochondrial clearance [90]. In this experiment, $A \beta$ did not cause the activation of BNIP3/NIX. In addition, Parkin overexpression in AD mouse models led to enhanced autophagic clearance of defective mitochondria and prevented mitochondrial dysfunction [91, 92]. In short, among the many different mitochondrial autophagic pathways, PINK1/Parkin-dependent mitochondrial autophagy is the focus of current AD research. We found that after si-CD36 treatment, $A \beta 1-40$-induced mitochondrial autophagy by PINK1/Parkin was blocked, indicating that $A \beta 1-40$ caused pericyte mitochondrial autophagy through the CD36/PINK1/Parkin pathway.

\section{Conclusion}

In summary, we discovered a new cell cascade response induced by A $\beta 1-40$ and identified pericyte CD36 as a potential target of intervention for the treatment of AD-induced BBB destruction, which will prevent and improve nerve damage. Degenerative diseases need new therapies. In the future, more translational studies are needed to evaluate the role and mechanism of regulation of mitochondrial autophagy and its induced iron death for the treatment of aging, neurodegeneration, and other diseases.

\section{Declarations}

\section{Ethics approval and consent to participate}

During the experiment, raising and obtaining animal materials complied with the relevant rules and regulations of the Animal Management Committee of Huazhong University of Science and Technology and the International Association for Animal Research (IASP) on the management and protection of experimental animals.

\section{Consent for publication}

All of the authors have approved the contents of this paper.

\section{Availability of data and material}

The datasets used and/or analyzed during the current study are available from the corresponding author on reasonable request. 


\section{Competing interests}

We declare no conflict of interest relevant to this review article.

\section{Funding}

This study was primarily supported by grants from National Natural Science Foundation of China (Grant No. 81801204).

\section{Authors' contributions}

CY designed the study and helped to review the manuscript. JL performed experiments, inter-preted the results, and wrote the manuscript; ML performed experiments, analyzed data, and helped to prepare the manuscript. YG performed experiments and analyzed data. JC performed experiments and interpreted the results. JM and CW performed experiments. MS and LW participated in the drawing. SY helped to interpret the results.

\section{Acknowledgments}

The authors would like to thank both the Institute of Anesthesia and Critical Care Medicine Laboratory of Union Hospital for supporting and all the lab colleagues for assistance. Also, the authors would like to thank Home for AJE (www.aje.cn) for their English language editing.

\section{References}

1. Sweeney, M.D., et al., Vascular dysfunction-The disregarded partner of Alzheimer's disease. Alzheimers Dement, 2019. 15(1): p. 158-167.

2. Sweeney, M.D., A.P. Sagare, and B.V. Zlokovic, Cerebrospinal fluid biomarkers of neurovascular dysfunction in mild dementia and Alzheimer's disease. J Cereb Blood Flow Metab, 2015. 35(7): p. 1055-68.

3. Montagne, A., et al., Blood-brain barrier breakdown in the aging human hippocampus. Neuron, 2015. 85(2): p. 296-302.

4. Montagne, A., et al., Brain imaging of neurovascular dysfunction in Alzheimer's disease. Acta Neuropathol, 2016. 131(5): p. 687-707.

5. Kisler, K., et al., Cerebral blood flow regulation and neurovascular dysfunction in Alzheimer disease. Nat Rev Neurosci, 2017. 18(7): p. 419-434.

6. Armulik, A., et al., Pericytes regulate the blood-brain barrier. Nature, 2010. 468(7323): p. 557-61.

7. Sweeney, M.D., S. Ayyadurai, and B.V. Zlokovic, Pericytes of the neurovascular unit: key functions and signaling pathways. Nat Neurosci, 2016. 19(6): p. 771-83.

8. Bell, R.D., et al., Pericytes control key neurovascular functions and neuronal phenotype in the adult brain and during brain aging. Neuron, 2010. 68(3): p. 409-27. 
9. Sengillo, J.D., et al., Deficiency in mural vascular cells coincides with blood-brain barrier disruption in Alzheimer's disease. Brain Pathol, 2013. 23(3): p. 303-10.

10. Farkas, E. and P.G. Luiten, Cerebral microvascular pathology in aging and Alzheimer's disease. Prog Neurobiol, 2001. 64(6): p. 575-611.

11. Baloyannis, S.J. and I.S. Baloyannis, The vascular factor in Alzheimer's disease: a study in Golgi technique and electron microscopy. J Neurol Sci, 2012. 322(1-2): p. 117-21.

12. Aguilera, K.Y. and R.A. Brekken, Recruitment and retention: factors that affect pericyte migration. Cell Mol Life Sci, 2014. 71(2): p. 299-309.

13. Nikolakopoulou, A.M., et al., Regional early and progressive loss of brain pericytes but not vascular smooth muscle cells in adult mice with disrupted platelet-derived growth factor receptor-beta signaling. PLoS One, 2017. 12(4): p. e0176225.

14. Erdő, F., L. Denes, and E. de Lange, Age-associated physiological and pathological changes at the blood-brain barrier: A review. J Cereb Blood Flow Metab, 2017. 37(1): p. 4-24.

15. Zenaro, E., G. Piacentino, and G. Constantin, The blood-brain barrier in Alzheimer's disease. Neurobiol Dis, 2017. 107: p. 41-56.

16. Bero, A.W., et al., Neuronal activity regulates the regional vulnerability to amyloid- $\beta$ deposition. Nat Neurosci, 2011. 14(6): p. 750-6.

17. Hardy, J.A. and G.A. Higgins, Alzheimer's disease: the amyloid cascade hypothesis. Science, 1992. 256(5054): p. 184-5.

18. Pantoni, L., Cerebral small vessel disease: from pathogenesis and clinical characteristics to therapeutic challenges. Lancet Neurol, 2010. 9(7): p. 689-701.

19. Tarasoff-Conway, J.M., et al., Clearance systems in the brain-implications for Alzheimer disease. Nat Rev Neurol, 2015. 11(8): p. 457-70.

20. Sagare, A.P., R.D. Bell, and B.V. Zlokovic, Neurovascular dysfunction and faulty amyloid $\beta$-peptide clearance in Alzheimer disease. Cold Spring Harb Perspect Med, 2012. 2(10).

21. Silverstein, R.L. and M. Febbraio, CD36, a scavenger receptor involved in immunity, metabolism, angiogenesis, and behavior. Sci Signal, 2009. 2(72): p. re3.

22. Febbraio, M., D.P. Hajjar, and R.L. Silverstein, CD36: a class B scavenger receptor involved in angiogenesis, atherosclerosis, inflammation, and lipid metabolism. J Clin Invest, 2001. 108(6): p. 785-91.

23. El Khoury, J.B., et al., CD36 mediates the innate host response to beta-amyloid. J Exp Med, 2003. 197(12): p. 1657-66.

24. Moore, K.J. and M.W. Freeman, Scavenger receptors in atherosclerosis: beyond lipid uptake. Arterioscler Thromb Vasc Biol, 2006. 26(8): p. 1702-11.

25. Park, L., et al., Innate immunity receptor CD36 promotes cerebral amyloid angiopathy. Proc Natl Acad Sci U S A, 2013. 110(8): p. 3089-94. 
26. Lemasters, J.J., Variants of mitochondrial autophagy: Types 1 and 2 mitophagy and micromitophagy (Type 3). Redox Biol, 2014. 2: p. 749-54.

27. Underly, R.G., et al., Pericytes as Inducers of Rapid, Matrix Metalloproteinase-9-Dependent Capillary Damage during Ischemia. J Neurosci, 2017. 37(1): p. 129-140.

28. Gonul, E., et al., Early pericyte response to brain hypoxia in cats: an ultrastructural study. Microvasc Res, 2002. 64(1): p. 116-9.

29. Iram, T., et al., Astrocytes from old Alzheimer's disease mice are impaired in A $\beta$ uptake and in neuroprotection. Neurobiol Dis, 2016. 96: p. 84-94.

30. Zachariah, M.A. and J.G. Cyster, Neural crest-derived pericytes promote egress of mature thymocytes at the corticomedullary junction. Science, 2010. 328(5982): p. 1129-35.

31. Venkataraman, K., et al., Vascular endothelium as a contributor of plasma sphingosine 1-phosphate. Circ Res, 2008. 102(6): p. 669-76.

32. Deane, R., et al., LRP/amyloid beta-peptide interaction mediates differential brain efflux of Abeta isoforms. Neuron, 2004. 43(3): p. 333-44.

33. Shibata, M., et al., Clearance of Alzheimer's amyloid-ss(1-40) peptide from brain by LDL receptorrelated protein-1 at the blood-brain barrier. J Clin Invest, 2000. 106(12): p. 1489-99.

34. Kimura, A., et al., Antagonism of sphingosine 1-phosphate receptor-2 enhances migration of neural progenitor cells toward an area of brain. Stroke, 2008. 39(12): p. 3411-7.

35. Keaney, J., et al., Autoregulated paracellular clearance of amyloid-beta across the blood-brain barrier. Sci Adv, 2015. 1(8): p. e1500472.

36. Morris, A.W., et al., The Cerebrovascular Basement Membrane: Role in the Clearance of $\beta$-amyloid and Cerebral Amyloid Angiopathy. Front Aging Neurosci, 2014. 6: p. 251.

37. Mizumura, K., et al., Mitophagy-dependent necroptosis contributes to the pathogenesis of COPD. J Clin Invest, 2014. 124(9): p. 3987-4003.

38. Givvimani, S., et al., Mitochondrial division/mitophagy inhibitor (Mdivi) ameliorates pressure overload induced heart failure. PLoS One, 2012. 7(3): p. e32388.

39. Yang, W.S. and B.R. Stockwell, Ferroptosis: Death by Lipid Peroxidation. Trends Cell Biol, 2016. 26(3): p. 165-176.

40. Koppula, P., L. Zhuang, and B. Gan, Cystine transporter SLC7A11/XCT in cancer: ferroptosis, nutrient dependency, and cancer therapy. Protein Cell, 2020.

41. Yang, W.S., et al., Regulation of ferroptotic cancer cell death by GPX4. Cell, 2014. 156(1-2): p. 317331.

42. Gao, M., et al., Ferroptosis is an autophagic cell death process. Cell Res, 2016. 26(9): p. 1021-32.

43. Stockwell, B.R., et al., Ferroptosis: A Regulated Cell Death Nexus Linking Metabolism, Redox Biology, and Disease. Cell, 2017. 171(2): p. 273-285.

44. Mawuenyega, K.G., et al., Decreased clearance of CNS beta-amyloid in Alzheimer's disease. Science, 2010. 330(6012): p. 1774. 
45. Sagare, A.P., R.D. Bell, and B.V. Zlokovic, Neurovascular defects and faulty amyloid- $\beta$ vascular clearance in Alzheimer's disease. J Alzheimers Dis, 2013. 33 Suppl 1(0 1): p. S87-100.

46. Deane, R., et al., RAGE mediates amyloid-beta peptide transport across the blood-brain barrier and accumulation in brain. Nat Med, 2003. 9(7): p. 907-13.

47. Kawarabayashi, T., et al., Age-dependent changes in brain, CSF, and plasma amyloid (beta) protein in the Tg2576 transgenic mouse model of Alzheimer's disease. J Neurosci, 2001. 21(2): p. 372-81.

48. Iadecola, C., The overlap between neurodegenerative and vascular factors in the pathogenesis of dementia. Acta Neuropathol, 2010. 120(3): p. 287-96.

49. Park, L., et al., Scavenger receptor CD36 is essential for the cerebrovascular oxidative stress and neurovascular dysfunction induced by amyloid-beta. Proc Natl Acad Sci U S A, 2011. 108(12): p. 5063-8.

50. Mosconi, L., et al., Reduced hippocampal metabolism in MCl and AD: automated FDG-PET image analysis. Neurology, 2005. 64(11): p. 1860-7.

51. Ferris, S.H., et al., Positron emission tomography in the study of aging and senile dementia. Neurobiol Aging, 1980. 1(2): p. 127-31.

52. Miners, J.S., I. Schulz, and S. Love, Differing associations between A $\beta$ accumulation, hypoperfusion, blood-brain barrier dysfunction and loss of PDGFRB pericyte marker in the precuneus and parietal white matter in Alzheimer's disease. J Cereb Blood Flow Metab, 2018. 38(1): p. 103-115.

53. Sagare, A.P., et al., Pericyte loss influences Alzheimer-like neurodegeneration in mice. Nat Commun, 2013. 4: p. 2932.

54. Wilhelmus, M.M., et al., Lipoprotein receptor-related protein-1 mediates amyloid-beta-mediated cell death of cerebrovascular cells. Am J Pathol, 2007. 171(6): p. 1989-99.

55. Tachibana, M., et al., Pericyte implantation in the brain enhances cerebral blood flow and reduces amyloid-beta pathology in amyloid model mice. Exp Neurol, 2018. 300: p. 13-21.

56. Iadecola, C., et al., SOD1 rescues cerebral endothelial dysfunction in mice overexpressing amyloid precursor protein. Nat Neurosci, 1999. 2(2): p. 157-61.

57. Zlokovic, B.V., The blood-brain barrier in health and chronic neurodegenerative disorders. Neuron, 2008. 57(2): p. 178-201.

58. Arvanitakis, Z., et al., Relation of cerebral vessel disease to Alzheimer's disease dementia and cognitive function in elderly people: a cross-sectional study. Lancet Neurol, 2016. 15(9): p. 934-943.

59. Yamazaki, Y. and T. Kanekiyo, Blood-Brain Barrier Dysfunction and the Pathogenesis of Alzheimer's Disease. Int J Mol Sci, 2017. 18(9).

60. Zhao, Z., et al., Establishment and Dysfunction of the Blood-Brain Barrier. Cell, 2015. 163(5): p. 1064-1078.

61. Son, N.H., et al., Endothelial cell CD36 optimizes tissue fatty acid uptake. J Clin Invest, 2018. 128(10): p. 4329-4342. 
62. Stewart, C.R., et al., CD36 ligands promote sterile inflammation through assembly of a Toll-like receptor 4 and 6 heterodimer. Nat Immunol, 2010. 11(2): p. 155-61.

63. Kiffin, R., U. Bandyopadhyay, and A.M. Cuervo, Oxidative stress and autophagy. Antioxid Redox Signal, 2006. 8(1-2): p. 152-62.

64. Palikaras, K., E. Lionaki, and N. Tavernarakis, Coordination of mitophagy and mitochondrial biogenesis during ageing in C. elegans. Nature, 2015. 521(7553): p. 525-8.

65. Fang, E.F., et al., Defective mitophagy in XPA via PARP-1 hyperactivation and NAD(+)/SIRT1 reduction. Cell, 2014. 157(4): p. 882-896.

66. Mizushima, N., T. Yoshimori, and B. Levine, Methods in mammalian autophagy research. Cell, 2010. 140(3): p. 313-26.

67. Sun, Y., et al., Beclin-1-Dependent Autophagy Protects the Heart During Sepsis. Circulation, 2018. 138(20): p. 2247-2262.

68. Jin, W.S., et al., Peritoneal dialysis reduces amyloid-beta plasma levels in humans and attenuates Alzheimer-associated phenotypes in an APP/PS1 mouse model. Acta Neuropathol, 2017. 134(2): p. 207-220.

69. Frieden, M., et al., Subplasmalemmal mitochondria modulate the activity of plasma membrane Ca2+ATPases. J Biol Chem, 2005. 280(52): p. 43198-208.

70. Parsons, M.J. and D.R. Green, Mitochondria and apoptosis: a quick take on a long view. F1000 Biol Rep, 2009. 1: p. 17.

71. Montagne, A., et al., Pericyte degeneration causes white matter dysfunction in the mouse central nervous system. Nat Med, 2018. 24(3): p. 326-337.

72. Cardaioli, E., et al., A new thymidine phosphorylase mutation causing elongation of the protein underlies mitochondrial neurogastrointestinal encephalomyopathy. J Neurol, 2012. 259(1): p. 172-4.

73. Garza-Lombó, C., et al., Redox homeostasis, oxidative stress and mitophagy. Mitochondrion, 2020. 51: p. 105-117.

74. Murphy, M.P., How mitochondria produce reactive oxygen species. Biochem J, 2009. 417(1): p. 1-13.

75. Giorgi, C., et al., Mitochondria and Reactive Oxygen Species in Aging and Age-Related Diseases. Int Rev Cell Mol Biol, 2018. 340: p. 209-344.

76. Angelova, P.R. and A.Y. Abramov, Role of mitochondrial ROS in the brain: from physiology to neurodegeneration. FEBS Lett, 2018. 592(5): p. 692-702.

77. Chen, X., et al., Ferroptosis: machinery and regulation. Autophagy, 2020: p. 1-28.

78. Tang, M., et al., Ferritinophagy/ferroptosis: Iron-related newcomers in human diseases. J Cell Physiol, 2018. 233(12): p. 9179-9190.

79. Seibt, T.M., B. Proneth, and M. Conrad, Role of GPX4 in ferroptosis and its pharmacological implication. Free Radic Biol Med, 2019. 133: p. 144-152.

80. Dixon, S.J. and B.R. Stockwell, The role of iron and reactive oxygen species in cell death. Nat Chem Biol, 2014. 10(1): p. 9-17. 
81. Matsuda, N., et al., PINK1 stabilized by mitochondrial depolarization recruits Parkin to damaged mitochondria and activates latent Parkin for mitophagy. J Cell Biol, 2010. 189(2): p. 211-21.

82. Fritsch, L.E., et al., Ubiquitin and Receptor-Dependent Mitophagy Pathways and Their Implication in Neurodegeneration. J Mol Biol, 2020. 432(8): p. 2510-2524.

83. Kondapalli, C., et al., PINK1 is activated by mitochondrial membrane potential depolarization and stimulates Parkin E3 ligase activity by phosphorylating Serine 65. Open Biol, 2012. 2(5): p. 120080.

84. Lazarou, M., et al., The ubiquitin kinase PINK1 recruits autophagy receptors to induce mitophagy. Nature, 2015. 524(7565): p. 309-314.

85. Morales, P.E., et al., Emerging role of mitophagy in cardiovascular physiology and pathology. Mol Aspects Med, 2020. 71: p. 100822.

86. Fivenson, E.M., et al., Mitophagy in neurodegeneration and aging. Neurochem Int, 2017. 109: p. 202209.

87. Schweers, R.L., et al., NIX is required for programmed mitochondrial clearance during reticulocyte maturation. Proc Natl Acad Sci U S A, 2007. 104(49): p. 19500-5.

88. Gao, A., et al., Bnip3 in mitophagy: Novel insights and potential therapeutic target for diseases of secondary mitochondrial dysfunction. Clin Chim Acta, 2020. 506: p. 72-83.

89. Chen, G., et al., Nix and Nip3 form a subfamily of pro-apoptotic mitochondrial proteins. J Biol Chem, 1999. 274(1): p. 7-10.

90. Liu, L., et al., Receptor-mediated mitophagy in yeast and mammalian systems. Cell Res, 2014. 24(7): p. 787-95.

91. Martin-Maestro, P., et al., PARK2 enhancement is able to compensate mitophagy alterations found in sporadic Alzheimer's disease. Hum Mol Genet, 2016. 25(4): p. 792-806.

92. Khandelwal, P.J., et al., Parkin mediates beclin-dependent autophagic clearance of defective mitochondria and ubiquitinated Abeta in AD models. Hum Mol Genet, 2011. 20(11): p. 2091-102.

\section{Figures}

\section{Figure 1}

The APP/PS1 mouse BBB is damaged, pericytes are reduced, and CD36 expression is reduced. pericytes express CD36 and colocalize with A 1 1. (A: PET/CT scan images of mice in each group.) Brain glucose metabolic images of the APP/PS1 mice and the WT mice (taken 0-1 min after 18F-FDG injection). B: Protein expression levels of PDGFR 3, NG2, CD36, and LRP1 in brain tissue. (C) Immunohistochemical $(\mathrm{IHC})$ staining shows the formation of amyloid plaques composed of $A \beta$. (D) Immunofluorescence showed that there were fewer APP/PS1 mouse endothelial cell (GLUT1 pink label)-covered pericytes (PDGFR $\beta$ green label) than those of the WT mice (10 areas of the cortex and hippocampus were used for 
image acquisition). (E) Immunofluorescence shows that CD36 (pink) and A $\beta$ (green) are colocalized in pericytes (red). Blue indicates DAPI. (\#\#p<0.01, \#\#\#p<0.001 compared with the WT group, $\Delta \Delta p<0.01$ compared with the APP/PS1 6 mo group, $n=6$ ).

\section{Figure 2}

$A \beta 1-40$ is transported to pericytes via CD36 and upregulates the expression of CD36 protein. (A) A 1-40 was incubated with pericytes for 1 hour, and the pericytes overlapped with red fluorescent $A \beta 1-40$ under an optical microscope. (B) Cellular immunofluorescence showed red fluorescent A 1-40 in the pericytes and CD36 (green light). Colocalization was observed. Blue indicates DAPI, scale $20 \mu \mathrm{m}$. (C) Western blotting was used to detect CD36 and LRP1 protein expression after 6 hours of treatment of pericytes with AB1-40 (100 nM and $1 \mu M)$. (D, E) qRT-PCR was used to detect the changes in the transcriptional levels of CD36 and LRP1 in AB1-40 (100 nM and $1 \mu \mathrm{M})$-treated pericytes for 6 hours. (F) Western blots were used to detect the expression of CD36 at the protein level after RNA interference. (G) qRT-PCR detects the expression changes induced by si-CD36 at the transcriptional level $(\# p<0.05, \# \# p<0.01$, $\# \# \# p<0.001$ compared with the CTR group, $\Delta \Delta p<0.01$ compared with the $100 \mathrm{nM} A \beta 1-40$ group, $n=3$ ). Note: si-NC indicates the control group.

\section{Figure 3}

$A \beta 1-40$ increases the permeability of the BBB, and downregulating the expression of CD36 can improve the BBB changes caused by A 1 1-40. (A) TEER detects the BBB tightness of AB1-40-treated cells (100 nM and $1 \mu \mathrm{M}$ ) in monolayer and coculture models for 6 hours. (B) TEER detects the tightness of the BBB between the $A \beta 1-40(100 \mathrm{nM}$ and $1 \mu \mathrm{M})$-treated pericyte si-CD36 coculture group and the control group after 6 hours [ ${ }^{*} p<0.05$ in $A$ and $B$ compared with the same group, $\# p<0.05$ compared with the BEC singlelayer model CTR group, $\triangle p<0.05$ compared with the BEC/PC (A) or BEC/PC si-NC (B) coculture model CTR group, $n=3$ ]. (C) The fluorescence microplate reader detects the normalized permeability of FITC-dextran after $1 \mu \mathrm{M} A \beta 1-40$ treatment of the coculture model. (D) The fluorescence microplate reader detects the normalized permeability of FITC-A $1-40$ after $1 \mu \mathrm{M}$ A 1-40 treatment of the coculture model. (\#\#p<0.01 vs. the $B E C / P C$ group, ${ }^{*} p<0.05$ vs. the BEC/PC si-NC group, $n=3$ ). Note: $B E C$ : $b E n d .3$ is cultured alone, BEC/PC: bEnd.3/pericytic coculture, si-NC: empty vector control.

\section{Figure 4}

Mitochondrial autophagic changes after 6 hours of treatment of pericytes with low-dose Aß1-40 (100 nM and $1 \mu \mathrm{M}$ ). (A) The CCK-8 assay was used to detect the proliferation of pericytes after stimulation with $A \beta 1-40$. (B, C) Flow cytometry was used to detect the effect of AB1-40 on mitochondrial damage after pericytes (MitoSOX: mitochondrial ROS, TMRM: mitochondrial membrane potential). (D) Western blotting 
was used to detect the expression changes of Tim23, HSP60, and LC3II/LC3I at the protein level. (E-F) qRT-PCR was used to detect the expression changes of Tim23 and HSP60 at the transcriptional level. (G) Immunofluorescence was used to detect the colocalization of autophagosomes (LC3 red) and mitochondria (HSP60 green). (H)Confocal microscopy with a lysosomal tracer (LysoTracker Red) and mitochondria marker (HSP60 green) was used to colocalize proteins. $\left({ }^{*} p<0.05,{ }^{*} p<0.01\right.$ compared with

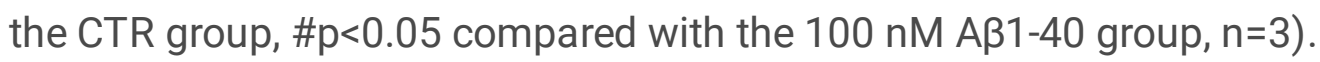

\section{Figure 5}

Autophagic agonists and inhibitors verified that $1 \mu \mathrm{M} A \beta 1-40$ treatment of pericytes led to increased mitochondrial autophagy and increases the generation of ROS. (A) Western blot detection of the mitochondrial proteins Tim23 and HSP60 and the autophagosome protein LC3II/LC3I at the protein level after $5 \mu \mathrm{M}$ CCCP treatment as a positive control. (B) Western blots to detect the expression changes of the mitochondrial proteins Tim23 and HSP60 at the protein level with or without $20 \mu \mathrm{M} \mathrm{CQ}$. (C) Western blot to detect the expression changes of mitochondrial proteins Tim23 and HSP60 at the protein level with or without Mdivi-1 $20 \mu \mathrm{M}$. ( ${ }^{\star \star} \mathrm{p}<0.01$ compared with the CTR group, \&\&p<0.01 compared with the 1 $\mu \mathrm{M}$ Aß1-40 group, $\mathrm{n}=3$ ). (D) Use of $5 \mu \mathrm{M}$ CCCP as a positive control and typical transmission electron micrographs after 6 hours with or without $20 \mu \mathrm{M}$ Mdivi-1. At least 6 cells were examined under each treatment condition. (E) Western blotting was used to detect changes in the expression of apoptosisrelated BCL2/BAX and P17/P35 CASP3 after AB1-40 (100 nM and $1 \mu \mathrm{M})$ stimulation of pericytes for 6 hours. (F) Flow cytometry to detect the effect of AB1-40 on pericyte apoptosis. (G) Fluorescence microscopy observation and detection of the fluorescent probe DHE indicate that A 1-40 stimulation changes ROS expression in pericytes. $(\mathrm{H})$ Fluorescence microscopy observation and detection of the fluorescent probe for ROS, which is used to show the changes in ROS expression in pericytes caused by A 1-40 stimulation. (I) The ATP kit detects the changes in pericyte energy produced by A 1-40 stimulation. $(\mathrm{J})$ SOD kit detection of the effect of $A \beta 1-40$ stimulation on the antioxidant capacity of pericytes ( ${ }^{*} p<0.05,{ }^{*} \mathrm{p}<<0.01$ and CTR group, $\mathrm{n}=3$ ).

\section{Figure 6}

A low dose of A 1-40 causes cellular mitophagy-dependent lipid peroxidation and induces ferroptosis through CD36/PINK1/Parkin pathway. (A) The GSH-PX kit was used to detect the changes in pericyte glutathione peroxidase stimulated by AB1-40. (B) The iron kit detects changes in iron ions. (C) A fluorescence microplate reader (Mito-FerroGreen) was used to detect the change in Fe2+. (D) Fluorescence microplate reader analysis of BODIPY581/591 detects the effect of lipid active oxygen. (E) Western blot analysis was used to detect changes in the expression of the iron death-related proteins GPx4, xCT, ferritin, and NOX1 after stimulation of pericytes with $1 \mu \mathrm{M} \mathrm{A \beta 1-40} \mathrm{or} 10 \mu \mathrm{M}$ erastin for 6 hours. (F) Western blotting was used to detect the changes in the expression of the iron death-related proteins GPx4, xCT, ferritin, and NOX1 after pericytes were stimulated with $1 \mu \mathrm{M}$ Aß1-40 with or without FER-1 for 6 
hours. $(\mathrm{H})$ Erastin $(10 \mu \mathrm{M})$ was used as a positive control; typical transmission electron micrograph after 6 hours with or without FER-1 $(10 \mu \mathrm{M})$. At least 6 cells were examined under each treatment condition. (I) Western blotting was used to detect the expression levels of the mitochondrial pathway proteins S65, BNIP3/NIX, and PINK1/Parkin after $1 \mu \mathrm{M}$ Aß1-40 treatment of pericytes for 6 hours. (J) Western blot detection of the effect of $1 \mu \mathrm{M}$ A 1-40 on the expression of CD36, PINK1/Parkin, HSP60, and Tim23 in pericytes 6 hours after si-CD36 transfection. ( ${ }^{\star} p<0.05$, ${ }^{\star \star} p<0.01,{ }^{\star \star \star} p<0.001$ compared with the CTR group, \&p<0.05, \&\&p<0.01 compared with the $A \beta 1-401 \mu M$ group, $n=3$ or 6$)$. 